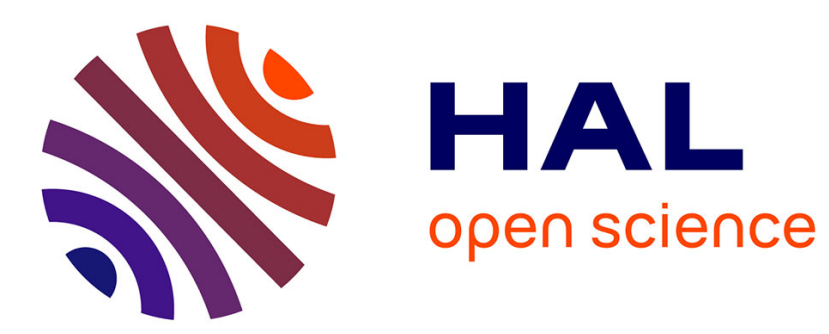

\title{
Rewriting Systems for Reachability in Vector Addition Systems with Pairs
}

\author{
Paulin Jacobé de Naurois, Virgile Mogbil
}

\section{To cite this version:}

Paulin Jacobé de Naurois, Virgile Mogbil. Rewriting Systems for Reachability in Vector Addition Systems with Pairs. 4th International Workshop on Reachability Problems (RP'10), Aug 2010, Brno, Czech Republic. pp. 133-145, 10.1007/978-3-642-15349-5 . hal-00483235v2

\section{HAL Id: hal-00483235 \\ https://hal.science/hal-00483235v2}

Submitted on 14 Jun 2010

HAL is a multi-disciplinary open access archive for the deposit and dissemination of scientific research documents, whether they are published or not. The documents may come from teaching and research institutions in France or abroad, or from public or private research centers.
L'archive ouverte pluridisciplinaire $\mathbf{H A L}$, est destinée au dépôt et à la diffusion de documents scientifiques de niveau recherche, publiés ou non, émanant des établissements d'enseignement et de recherche français ou étrangers, des laboratoires publics ou privés. 


\title{
Rewriting Systems for Reachability in Vector Addition Systems with Pairs
}

(rapport interne LIPN - May 2010)

\author{
Paulin Jacobé de Naurois and Virgile Mogbil \\ LIPN, UMR CNRS 7030 - Université Paris 13, \\ Institut Galilée, 99, avenue Jean-Baptiste Clément, 93430 Villetaneuse, France
}

\begin{abstract}
We adapt hypergraph rewriting system to a generalization of Vector Addition Systems with States (VASS) that we call vector addition systems with pairs (VASP). We give rewriting systems and strategies, that allow us to obtain reachability equivalence results between some classes of VASP and VASS. Reachability for the later is well known be equivalent to reachability in Petri nets. VASP generalize also Branching Extension of VASS (BVASS) for which it is unknown if they are more expressive than VASS. We consider here a more restricted notion of reachability for VASP than that for BVASS. However the reachability decision problem corresponding is already equivalent to decidability of the provability in Multiplicative and Exponential Linear Logic (MELL), a question left open for more than 20 years.
\end{abstract}

\section{Introduction}

Vector Addition Systems with Pairs (VASP) are Vector Addition Systems with States (VASS) extended with paired arcs. A VASS is a directed graph whose arcs are labeled by vectors in $\mathbb{Z}^{m}$, and nodes are used as control states. Vector addition is done when traversing an arc, starting from a value associated to the source state and producing a value associated to the target state. A VASP is a VASS, together with a set of disjoint arc pairs, where two arcs of a pair share the same source or the same target. The configurations of a VASP are multisets of vectors (values) in $\mathbb{Z}^{m}$, and its operational semantics is the following: when traversing an arc pair with shared source the value of the source state is split, and when the target is shared the values of the two sources are added. While VASS are well known to be equivalent to Petri Nets, VASP correspond to Petri Nets with split and join transitions, where a multiset of markings evolves accordingly to the transitions fired, the size of this multiset being increased or decreased when firing a split or join transition respectively.

VASP are defined as a restriction of directed hypergraph. They inherit notions of paths in hypergraph and connectedness [5], but the chosen presentation as paired graph allows us to keep also usual paths in underlying graphs without pairs (in other words paths using part of hyperarcs). Hypergraph rewriting systems give rewriting systems for VASP. Yet, (hyper- and) graph rewriting termination in general is undecidable [16]. In order to obtain termination, we look 
at rewriting strategies for VASP, which we use to rewrite some classes of VASP as VASS, and to transfer VASS reachability results to these classes of VASP.

Branching Extension of VASS (BVASS also called VATA [2]) for which KarpMiller trees were designed [18] are by the way a particular restriction of VASP, where arcs in a pair do only share their target. A motivating result about BVASS was given by [2]: the reachability decision problem for BVASS is equivalent to the open decision problem of the provability in Multiplicative and Exponential Linear Logic (MELL [8]). The VASP operational semantic we consider is restricted, so we have not full generalization of BVASS but an essential intersection. In fact we allow to traverse pairs only under a condition called division of values, that preserves the sign component-wise. However our condition is implicit for reachability problems with positive values, like in the proof given by [2] to establish their equivalence result.

Originally VASP were designed by the first author (work in progress) to work on the provability decidability in MELL. The second author gives here a slightly different presentation with explicit paths to study them by rewriting systems. We think that VASP allow to work on an adaptation of the technical proof of the reachability problem decidability for Petri nets $[11,13,17,12,4]$, with the goal to obtain the reachability decidability for Petri nets with split and join transitions. This is a work in progress not presented here, which may imply the decidability of the provability in MELL.

Outline. We present in the first section basic definitions of VASP and ribbons, which are paths in VASP. VASP model which generalizes VASS need to introduce new material and redefine both the notions of paths and reachability. Then we give the operational semantic. It is made through promenades which give the reachable values in a VASP. Reachability decision problem is defined in this subsection.

The second section is devoted to rewriting systems for VASP. The definitions are standard in rewriting systems for hyper-graphs. We present rewriting systems and rules as a tool for reachability study. An important subsection contains the set of rules we use and our key lemma of reachability preservation when rules are applied. We also describe a limited strategy for ribbons.

In the last section we give results of reachability relationships between VASP and VASS. There is two kind of results: lemmas about reachability preservation by rewriting strategies, and corollaries about reduction of reachability decision problem for certain classes of VASP to reachability decision problem for VASS.

\section{VASP And Reachability}

\subsection{Basic definitions}

Given $m>0$, values are vectors in $\mathbb{Z}^{m}$. If not precised, operations done on vectors are component-wise. Components of a vectors are given by the projections $(x)_{j}$ for $1 \leqslant j \leqslant m$. A value $x \in \mathbb{Z}^{m}$ is divided into $\left(x_{1}, x_{2}\right)$ when $x=x_{1}+x_{2}$ and $\forall 1 \leqslant j \leqslant m,\left|(x)_{j}\right|=\left|\left(x_{1}\right)_{j}\right|+\left|\left(x_{2}\right)_{j}\right|$. Remark that positive components of a value are divided into positive integers. 
Lemma 1. Given $m>0$, let $x \geqslant y$ be values in $\mathbb{Z}^{m}$. If $\left(y_{1}, y_{2}\right)$ is a division of $y$ then there exists a division $\left(x_{1}, x_{2}\right)$ of $x$ such that $x_{1} \geqslant y_{1}$ and $x_{2} \geqslant y_{2}$.

Definition 1 (VASP). A Vector Addition System with States (VASS) $(m, G, v)$ of dimension $m>0$ is a directed graph $G=(Q, A)$ whose vertices are called states, together with a labeling function $v: A \mapsto \mathbb{Z}^{m}$, called valuation, that associates a value to each arc in $A$. For a given arc $a=(s, t) \in Q^{2}$, we call source $S(a)=s$ and target $T(a)=t$ respectively the source state and the target state of a.

$A$ Vector Addition System with Pairs (VASP) $(m, G, P, v)$ of dimension $m$ is a vector addition system with states $(m, G, v)$ whose graph $G=(Q, A)$ is equipped with a set $P \subseteq A \times A$ of disjoint pairs, such that the two arcs of each pair in $P$ share the same source or the same target, called a paired state. A source or target sharing pair (resp. shared arc) is called a split or join pair respectively (resp. split or join arc). An arc is called regular if it does not occur in the pair set. A paired graph $(G, P)$ is a graph $G$ equipped with such a pair set $P$. A paired graph $\left(G^{\prime}, P^{\prime}\right)$ is a subgraph of $(G, P)$ if and only if $P^{\prime} \subseteq P$ and $G^{\prime}$ is a subgraph of $G$ such that no arc in $G^{\prime} \backslash P^{\prime}$ is paired in $P$. A sub-VASP of a VASP $V$ is a $V A S P$ of same dimension and valuation whose paired graph is a subgraph of that of $V$. The reverse of a VASP $V$, denoted $V^{\text {rev }}$, is a VASP with same dimension, valuation, states and paired states, obtained by reversing arc orientations.

Remark that VASS are VASP with empty pair set. Without lost of generality, we consider VASP of dimension $m$ where all paired arcs are valued with the null vector denoted $0^{m} .1$

Definition 2 (configuration). A single configuration of a $\operatorname{VASP}(m, G=$ $(Q, A), P, v)$ is a couple $(q, x) \in Q \times \mathbb{Z}^{m}$. A configuration of $(m, G, P, v)$ is a multiset of single configurations. A configuration of $(m, G, P, v)$ is positive when the value of any of its single configurations is positive. A configuration of $(m, G, P, v)$ is divisible if the set of contained values is a division of its sum.

With the generalization to paired graphs, there are multiple kinds of paths. We keep the simplest one from graphs but the notion of cycle is generalized. E.g. $(2,4,3)$ is a cycle from 2 to 3 in Fig. 1(b).

Definition 3 (path). A path in a $\operatorname{VASP}(m, G=(Q, A), P, v)$ is a path in the graph $G$. We say that $(m, G, P, v)$ is weakly connected if $G$ is connected when orientation is removed. We say that $t$ is connected to $s$ in $(m, G, P, v)$ if there is a path from s to $t$ in $G$. Given a path $p$ in $(m, G, P, v), \bar{p}$ is the minimal subgraph of $(G, P)$ containing $p$. We say that a path $p$ from $s=S(a) \in \bar{p}$ to $t=S(b) \in \bar{p}$ is $a$ cycle if $a=b$ or $\{a, b\}$ is a pair in $\bar{p}$. In a simple path all arcs are distinct.

This notion of path in graphs is extended to VASP in the following way: in our paired graphs, a path is split and joined accordingly to paired arcs. We call ribbons such paths, by analogy with a (two sides) ribbon of paper cut with scissors (and sometimes paste). In order to have good properties over reversibility, ribbons are based on the notion of B-paths for which each source state must be reachable before the arc is traversable (Fig. 1(a)).

\footnotetext{
1 This will be clarified with the reachability definition.
} 


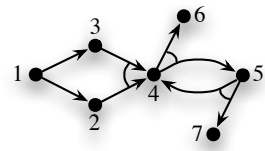

(a) A B-path

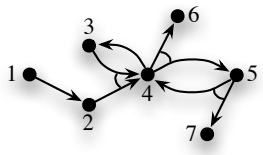

(b) Not a B-path from $\{1\}$

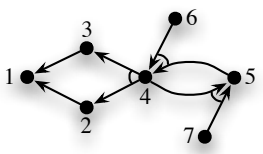

(c) Reverse of Fig. 1(a) VASP

Fig. 1. Examples

Definition 4 (ribbon). Given two sets of states $S$ and $T$, a Backward-path (or B-path) $B$ from $S$ to $T$ in a VASP $V$ is a minimal weakly connected sub$V A S P$ of $V$ with state set $Q^{\prime}$ such that i) $S, T \subseteq Q^{\prime}$, ii) if $q \in Q^{\prime}$ then $q$ is connected in $B$ to a state $s \in S$ by means of an acyclic simple path.

$A$ Forward-path (or F-path) from $S$ to $T$ in a VASP is a B-path from $T$ to $S$ in the reversed VASP. A ribbon (or BF-path) from $S$ to $T$ in a VASP is both a B-path and a F-path from $S$ to $T$. A state $t$ is B-connected (respectively Fconnected, BF-connected) to state $s$ if there exists a B-path (respectively F-path, $B F$-path) from $\{s\}$ to $\{t\}$. A bridge in a ribbon $\rho$ from $S$ to $T$ is a regular arc or an arc pair which disconnects $\rho$ into two or three connected components when it is removed, such that each of them gives a partition of $S$ or $T$ but not of both.

Paths in VASP subsume paths in graphs whereas B-path, F-path and BFpath are paths in hypergraph considering that paired arcs are hyperarcs. E.g. the VASP in Fig. 1(b) is not a B-path from $\{1\}$ because the only path connecting 3 to 1 contains the cycle $(2,4,3)$. However the cycle $(4,5,4)$ in Fig. 1 (a) is not contained in any simple path from 1 to 7 . See Fig. 2 for ribbon examples.

Proposition 1 ([5]). Given a B-path from $\{s\}$ containing an arc pair $\{a, b\}$, states $S(a)$ and $S(b)$ are $B$-connected to state $s$.

\section{$2.2 \quad$ Reachability}

When starting from a positive configuration $\left\{\left(s, x_{s}\right)\right\}_{s \in S}$ of a VASP, values reached following a ribbon from $S$ are given by a promenade:

Definition 5 (promenade, reachability). Given a VASP $V=(m, G, P, v)$ with a positive configuration $S$, a promenade $p$ on $V$ from $S$ is a sequence of configurations $\left(\mathcal{C}_{i}\right)_{i=0, \cdots, f}$ associated to a ribbon $\rho$ in $V$ from states of $S$ such that the initial configuration of $p$ is $\mathcal{C}_{0}=S$ and,

- given $c=(q, x) \in \mathcal{C}_{i}$ and a regular arc $a=\left(q, q^{\prime}\right) \in \rho$, then $\mathcal{C}_{i+1}=\mathcal{C}_{i}-\{c\} \cup$ $\left\{c^{\prime}\right\}$ where $c^{\prime}=\left(q^{\prime}, x+v(a)\right)$,

- given $c_{1}=\left(q_{1}, x_{1}\right) \in \mathcal{C}_{i}$ and $c_{2}=\left(q_{2}, x_{2}\right) \in \mathcal{C}_{i}$ and a join pair of $\rho\left(q_{1}, q^{\prime}\right)$ and $\left(q_{2}, q^{\prime}\right)$, then $\mathcal{C}_{i+1}=\mathcal{C}_{i}-\left\{c_{1}, c_{2}\right\} \cup\left\{c^{\prime}\right\}$ where $c^{\prime}=\left(q^{\prime}, x^{\prime}\right)$, and $\left(x_{1}, x_{2}\right)$ is a division of $x^{\prime}(\star)$,

- given $c=(q, x) \in \mathcal{C}_{i}$ and a split pair of $\rho\left(q, q_{1}\right)$ and $\left(q, q_{2}\right)$, then $\mathcal{C}_{i+1}=\mathcal{C}_{i}-$ $\{c\} \cup\left\{c_{1}^{\prime}, c_{2}^{\prime}\right\}$ where $c_{1}^{\prime}=\left(q_{1}, x_{1}\right), c_{2}^{\prime}=\left(q_{2}, x_{2}\right)$ and $x$ is non-deterministically divided into $\left(x_{1}, x_{2}\right)$.

A promenade from $S$ to the final configuration $T$ is a promenade associated to a ribbon from states of $S$ to states of $T$ such that division conditions are valid. $A$ 
positive promenade on a VASP is a promenade whose configurations are positive. We say that the final configuration $\mathcal{C}_{f}$ of a positive promenade on $V$ is reachable from the initial one $\mathcal{C}_{0}$. We denote it by: $\quad \mathcal{C}_{0} \rightsquigarrow V \mathcal{C}_{f}$.

Remark that our strong definition of both ribbons and division conditions for arc pairs in promenades imply that $\mathcal{C}_{0} \rightsquigarrow{ }_{V} \mathcal{C}_{f} \Leftrightarrow \mathcal{C}_{f} \rightsquigarrow V^{\text {rev }} \mathcal{C}_{0}$. Remark also that ribbons in a VASS coincide with paths, and a promenade (respectively a positive promenade) on a VASS $V$, considering it as a VASP with empty pair set, is then a sequence of single configurations (respectively with values in $\mathbb{N}^{m}$ ) associated to a path in $V$. So the classical reachability decision problem for VASS coincides with the one for VASP restricted to an empty pair set:

Reachability decision problem for VASP, denoted $\mathcal{R} \mathcal{P}_{\mathrm{VASP}}$ :

Input: Given a VASP $V$, two sets of single configurations $S$ and $T$ of $V$,

Question: Is there a positive promenade on $V$ between configurations whose underlying sets are $S$ and $T$ respectively?

Remark that contrary to BVASS case, $\mathcal{R} \mathcal{P}_{\mathrm{VASP}}$ is equivalent to a simpler restriction: Given a VASP $V$ and two configurations $S$ and $T$ of $V$, is there a positive promenade on $V$ between $S$ and $T$ ?

Variation. Branching VASS (BVASS) are VASP where we allow only regular arcs and join arcs. Compared to the operational semantic of BVASS, the operational semantic of VASP is restricted by the division condition for arc pairs in promenades, denoted $(\star)$ in the definition. Nevertheless the reachability decision problem for VASP without split arcs is equivalent to $\mathcal{R} \mathcal{P}_{\text {BVASS }}$. Indeed, in both cases, promenades are positive. It turns out that $\mathcal{R} \mathcal{P}_{\text {VASP }}$ implies $\mathcal{R} \mathcal{P}_{\text {BVASS }}$, which is equivalent to the decidability of MELL provability [2].

\section{VASP rewriting systems}

In this section we establish results concerning VASP reachability. For this purpose, we consider different restrictions of VASP, such as the separated ribbon (Figure 2).

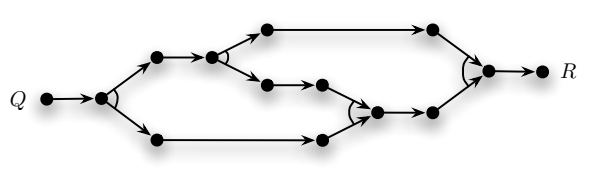

(a) A standard case

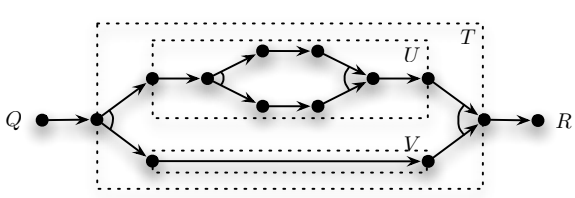

(b) An inductive case

Fig. 2. Examples of separated ribbons

Definition 6 (Separated ribbon). A 2-tree with root $s$ and leave set $L$ is a ribbon from $\{s\}$ to $L$ which contains no join arc and whose underlying graph is a rooted binary tree. A separated ribbon from $\{s\}$ to $\{t\}$ (Fig. 2) is a ribbon whose paired graph consists of a 2-tree with root $s$ and leaves set $L$, and a reversed 2-tree from $t$ to $L$. An inductive ribbon is a sequence of regular arcs, or is a 
separated ribbon that consists of 1) two disjoint inductive ribbons (like $U, V$ in Fig. 2(b)), related by 2) a sequence of regular arcs which finishes by a split pair, and by 3) a join pair which finishes by a sequence of regular arcs.

The separation refers to the set of arc pairs that is separated accordingly to split pairs and join pairs. In an inductive ribbon we can "glue" the sub-ribbons and then reduce reachability to VASS reachability:

Lemma 2. Let $V$ be a inductive ribbon from $\{s\}$ to $\{t\}$ with valuation $v$. Let $W$ be a VASP of same dimension which is only a regular arc $\left(s^{\prime}, t^{\prime}\right)$ valued by $\Sigma v(a)$ for all regular arc $a \in V$. We have:

$$
\left\{\left(s^{\prime}, x\right)\right\} \rightsquigarrow W\left\{\left(t^{\prime}, y\right)\right\} \quad \Rightarrow \quad\{(s, x)\} \rightsquigarrow V\{(t, y)\} .
$$

Proof. Remark that by definition of inductive ribbons we have equality of the underlying binary subtree heights. Given $m>0$, we define an inductive ribbon $S_{U, V}$ of dimension $m$ inductively on $\operatorname{size}\left(S_{U, V}\right)=1+\max \{\operatorname{size}(U), \operatorname{size}(V)\}$, where $U, V$ are the two disjoint inductive ribbons of the definition (like in Fig. 2(b)). The result is obtained by strong induction on this size.

Corollary 1. The reachability problem for inductive ribbon reduces to the reachability problem for VASS (via many-one reductions).

\subsection{Rewriting system definitions}

We generalize the previous approach by using rewriting systems for VASP as a tool for reachability proofs. We present rewriting systems as a restriction of hypergraph rewriting system. The goal is to give reachability results between VASP and rewritten VASP depending on rewriting systems and chosen strategies. As consequence, when we rewrite a VASP as a VASS, we have transfer reachability results from VASS to VASP.

Definition 7 (Morphism). A morphism $f: V \rightarrow V^{\prime}$ between two VASP $V=(m, G=(Q, A), P, v)$ and $V^{\prime}=\left(m, G^{\prime}=\left(Q^{\prime}, A^{\prime}\right), P^{\prime}, v^{\prime}\right)$ consists of two functions $f_{q}: Q \rightarrow Q^{\prime}$ and $f_{a}: A \rightarrow A^{\prime}$ preserving arcs, paired states and valuations. Such a morphism is an isomorphism if the functions $f_{q}$ and $f_{a}$ are bijective. In this case $V$ and $V^{\prime}$ are isomorphic, which is denoted by $V \simeq V^{\prime}$.

Definition 8 (Rule, Rewrite step). $A$ rule $r=(m, L \supseteq K \subseteq R)$ is a triple of $V A S P$ of dimension $m$ such that $K$ is a sub-VASP of both $L$ and $R$. The VASP $L$ and $R$ are called the left- and right-hand side of $r$, and $K$ is the interface.

Given a VASP $V$ of dimension $m$ and a set of rules $\mathcal{R}$, there is a rewrite step from $V$ based on $\mathcal{R}$ if there is a rule $r=(m, L \supseteq K \subseteq R)$ in $\mathcal{R}$ and a morphism $f: L \rightarrow V$ satisfying the following conditions: No arc in $V-f(L)$ is incident to any state in $f(L)-f(K)$, and for all distincts items $x, y \in L$, $f(x)=f(y)$ only if $x, y \in K$. We called $f(L)$ a redex for the rule $r$. The result (or reduct) of such a rewrite step is isomorphic to the VASP $W$ of dimension $m$ constructed as follows: let $U$ be the sub-VASP of $V$ obtained by removing all arcs and states in $f(L)-f(K)$ and by restricting the valuation of $V$, let $W$ be obtained from $U$ by adding disjointly all arcs, states and valuations in $R-K$. 
Rewrite steps from $V$ to $W$ based on $\mathcal{R}$ define the relation $\rightarrow \mathcal{R}$ between $V$ and $W$. We denote $\rightarrow_{\mathcal{R}}^{*}$ the transitive and reflexive closure of $\rightarrow_{\mathcal{R}}$, and relation sequences denote the composition. We abusively denote a rewriting rule $r=$ $(m, L \supseteq K \subseteq R)$ as a rewrite step from the left-hand side $L \rightarrow_{\{r\}} R$ where valuation is given, since $K$ is clear from the context.

Definition 9 (Rewriting system). Given some $n \geqslant 0$, a rewriting of length $n$ from $V$ to $V^{\prime}$ based on $\mathcal{R}$ is a sequence of the form $V \simeq V_{0} \rightarrow_{\mathcal{R}} V_{1} \rightarrow_{\mathcal{R}}$ $\cdots \rightarrow \mathcal{R} V_{n}=V^{\prime}$. A rewriting system $(m, \mathcal{R})$ is an integer $m>0$ and a finite set $\mathcal{R}$ of rules of dimension $m$. A rewriting system $(m, \mathcal{R})$ is terminating if there is not an infinite sequence of rewrite steps based on $\mathcal{R}$. Given a rewriting system $(m, \mathcal{R})$, a VASP $V$ of dimension $m$ is in normal form if there is not a VASP $W$ such that $V \rightarrow_{\mathcal{R}} W$, and $V$ has a normal form if there is a finite sequence from $V$ to some normal form.

\subsection{Our Rewriting Rules and Strategies}

When a rule interface is only a set of states, we give it by states labelled with capital letters $Q, R, S, \ldots$. Given $m>0$, we consider rules given in Fig. 3 called Regular Sum (RSum), Backward Zip (BZip), Backward Swap (BSwap), Collapse (Col), Zed (Zed) and Backward Expansion (BExp). For each rule, valuations are divided: that is, the valuation (of the regular arcs) of one side is a division of the valuation (of the regular arcs) of the other side. So they are set of rules. Abusively we consider rule sub-cases with the same name: BZip where one of the left-hand side regular arc is erased (and the corresponding source and target are merged), and BExp without regular arcs both side (then corresponding source and target are merged). We define rules called Forward Zip (FZip), Forward Swap (FSwap) and Forward Expansion (FExp) by respectively BZip, BSwap and BExp on the reversed VASP. We denote $r^{-1}$ the reversed relation of rule $r: r^{-1}$ goes from right-hand side of rule $r$ to left-hand side of rule $r$.

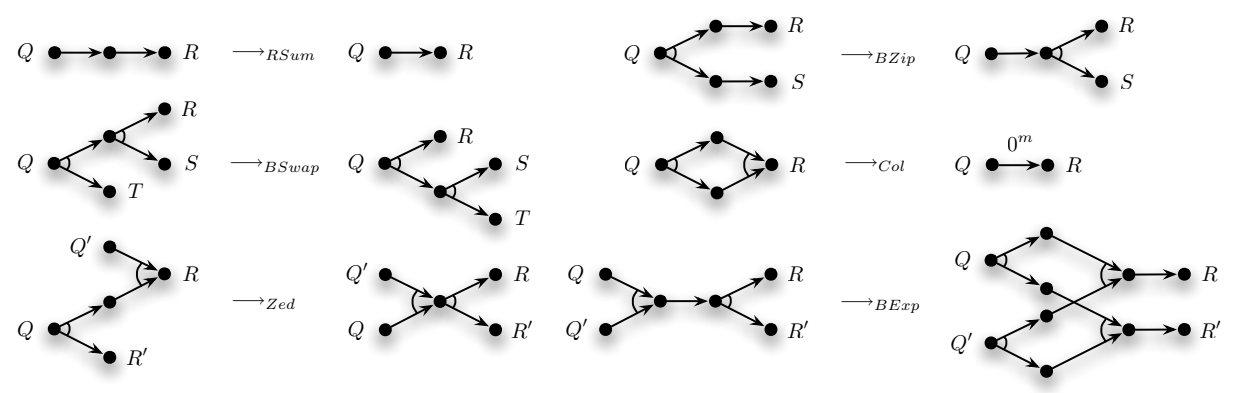

Fig. 3. Rewriting rules.

Remark that $Z e d, C o l$ and $R S$ um rules are their own reversed. Remark that by definition of rules and rewrite steps, there is no arc other than those indicated, to or from states which are not in a rule interface. 
Lemma 3 (Key lemma). Let $m>0$, let $r=(m, L \supseteq K \subseteq R)$ be one of the previously defined rules except $Z$ ed, but including reversed and inverted rules. Let $\mathcal{C}$ be a configuration of zero in-degree states of $L$ (labelled $Q, Q^{\prime}$ for Backward rules in Fig.3). Let $\mathcal{C}^{\prime}$ be a configuration of zero out-degree states of $R$. Then,

$$
\mathcal{C} \rightsquigarrow_{L} \mathcal{C}^{\prime} \quad \Leftrightarrow \quad \mathcal{C} \rightsquigarrow_{R} \mathcal{C}^{\prime} .
$$

Proof. Let $r \in \mathcal{R}$. Since by definition $\mathcal{C}$ and $\mathcal{C}^{\prime}$ are configurations of the interface of rule $r$, they are configurations of $R$. The result is easily obtained by a case analysis because of a division of a split pair is the "reverse" of a division of a join pair, and reciprocally. Notably, the result is obvious for BSwap, $\mathrm{Col}$ and $R S u m$, for all reversed Backward rules, and for inverse rules when proved for the corresponding rule. An interesting case is for the BZip rule: the right implication is clear. For the converse let us denote by $c$ the regular arc of $R$, and $a$ and $b$ the regular arcs of $L$. By definition of BZip we have a divided valuation: $(v(a), v(b))$ is a division of $v(c)$. Let $C=\{(Q, x)\}$, if $\mathcal{C} \rightsquigarrow R \mathcal{C}^{\prime}$ then $x \geqslant v(c)$ in $R$, and for any division $\left(x_{1}, x_{2}\right)$ of $x-v(c)$ we have $C^{\prime}=\left\{\left(R, x_{1}\right),\left(S, x_{2}\right)\right\}$. By lemma 1 let us take a division $\left(x_{1}, x_{2}\right)$ of $x-v(c)$ such that $x_{1} \geqslant v(a)$ and $x_{2} \geqslant v(b)$. This is the requirement for reachability from $C$ in $L$ : we obtain $\mathcal{C} \rightsquigarrow_{L} \mathcal{C}^{\prime} \Leftarrow \mathcal{C} \rightsquigarrow_{R} \mathcal{C}^{\prime}$.

For BZip rules, it is important to understand that only the requirement of divided valuation in VASP gives the left implication. Remark that only $\mathcal{C} \rightsquigarrow_{L}$ $\mathcal{C}^{\prime} \Rightarrow \mathcal{C} \rightsquigarrow_{R} \mathcal{C}^{\prime}$ holds for rule Zed.

From a reachability point of view, we cannot use a rewriting system without knowing if it is terminating, but (hyper- and) graph rewriting termination is undecidable [16]. Here we present a notion of strategies for a rewriting system that allows us to study termination of rewriting systems in a restricted case. Other methods are certainly useful but we are just interested in reachability. A strategy for a rewriting system is a VASP transformation that defines when a rewrite step is performed and what rule it takes:

Definition 10 (Strategy). Given a VASP rewriting system $\mathcal{S}$, a strategy is a function $f_{\mathcal{S}}$ from VASP set to itself. A strategy $f_{\mathcal{S}}$ is normalizing if whenever $V$ has a normal form, then there is some $n$ so that $f_{\mathcal{S}}{ }^{n}(V)$ is a normal form.

Given a rewriting system with a singleton rule set $\{r\}$, a strategy is usually a function that maps a VASP $V$ with a redex into $f_{\mathcal{S}}(V)$, the corresponding reduct obtained by one rewrite step from $V$. Sharper strategies give an order on the redexes for determining which redex is rewritten. A strategy implementation is often an algorithm for VASP traversal with a decision function to choose rules to be applied. We use in what follows this set of rules: $\mathcal{R}_{2}=\left\{\right.$ RSum, BZip ${ }^{-1}$, FZip $^{-1}$, BSwap, FSwap, BExp $\}$.

We consider the following separation strategy using $\mathcal{R}_{2}$ rules, which is a recursive function that maps a VASP $V$ with a ribbon $\rho$ to an isomorphic VASP where $\rho$ is rewritten into a separated ribbon by pushing away every split arcs to the left of join arcs (or the converse, from join arcs pushed away to the right, or by mixing them). BExp rule allows to cross in the right direction two arc pairs with 
"opposite" sharing. We are interested in terminaison of the separation strategy, but the BExp rule may create new BExp redexes. However we have:

Lemma 4. Given a rewriting system ( $m,\{B E x p\})$, there is no infinite sequence of rewrite steps in a ribbon.

Proof. We give a sketch by generalizing VASP pairs of paired graphs to hyperarcs of hypergraphs. In this case there is a measure that decreases in every BExp redex context, so terminaison for this rewrite rule generalizes to hyperarcs. This give us a bound on the number of $B E x p$ rewrite steps in the VASP by simulating with a fixed maximal number of steps the rule for hyperarcs.

\section{Reachability Relationship Between VASP and VASS}

We give a simple example illustrating how rewriting systems are used as a tool to obtain a reachability equivalence. With VASP rewriting system we just need to consider an ad-hoc strategy to rewrite a separated ribbon into a VASS.

Lemma 5. Let $V$ be a separated ribbon from $\{s\}$ to $\{t\}$ with valuation $v$. Let $W$ be a VASP of same dimension which is only a regular arc $\left(s^{\prime}, t^{\prime}\right)$ valued by $\Sigma v(a)$ for all regular arc $a \in V$. We have:

$$
\{(s, x)\} \rightsquigarrow V\{(t, y)\} \quad \Leftrightarrow \quad\left\{\left(s^{\prime}, x\right)\right\} \rightsquigarrow W\left\{\left(t^{\prime}, y\right)\right\}
$$

Proof. Let $V$ be a separated ribbon from $\{s\}$ to $\{t\}$ of dimension $m>0$ and valuation $v$. Let $W$ be the regular arc defined in the lemma hypothesis. Let $\mathcal{V}=(m,\{R S u m, C o l, B Z i p, F Z i p\})$ be a rewriting system. Let $U$ be the normal form of $V$ obtained by the following strategy on $\mathcal{V}$ : firstly from $s$ we apply $\overrightarrow{\mathcal{F}}=\left(\rightarrow_{\text {RSum }}^{*} \rightarrow_{\text {BZip }}\right)^{*}$ on the 2 -tree with root $\{s\}$, and we apply from $t$ the same reversed strategy, that is $\overleftarrow{\mathcal{F}}=\left(\rightarrow_{\text {RSum }}^{*} \rightarrow{ }_{F Z i p}\right)^{*}$, on the reversed 2-tree with root $\{t\}$. Secondly we apply $\rightarrow_{C o l}^{*}$ and we finish with $\rightarrow_{R S u m}^{*}$.

Corollary 2. The reachability problem for separated ribbon between states reduces to the reachability problem for VASS (via many-one reductions).

Now we give an example using a separation strategy which preserves reachability.

Lemma 6. Let $V$ be a ribbon from $\{s\}$ to $\{t\}$. Let $\mathcal{V}=\left(m, \mathcal{R}_{2}\right)$ be a rewriting system. There is separating strategy for $\mathcal{V}$ rewriting $V$ into a separated ribbon $W$ from $\left\{s^{\prime}\right\}$ to $\left\{t^{\prime}\right\}$ such that: $\quad\{(s, x)\} \rightsquigarrow_{V}\{(t, y)\} \Leftrightarrow\left\{\left(s^{\prime}, x\right)\right\} \rightsquigarrow W\left\{\left(t^{\prime}, y\right)\right\}$

Proof. Let $V$ be a ribbon from $\{s\}$ to $\{t\}$. Let the separation strategy be:

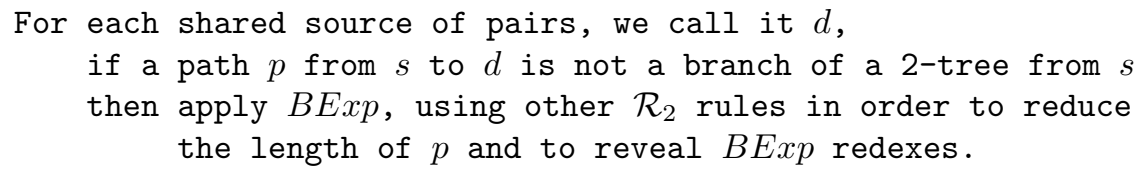


Remark that if there is a subpath $p^{\prime}$ of $p$ from a shared target of a pair to a $d$ state, then its length can always be reduced to zero by applying rules of $\mathcal{R}_{2}-\{B E x p\}$. It follows that in a not separated ribbon, BExp redexes can always be revealed. If the $d$ states to treat are chosen with smallest distance from $s$ then by lemma 4 the strategy terminates. Remark that $\mathcal{V}$ rewrites a ribbon into a ribbon. So $V$ is rewritten into a ribbon such that every shared source paired states are in a branch of a 2-tree from $s$. In other words this ribbon is separated.

Remark that a ribbon is defined to be both B-path and F-path, and this is essential to ensure that the strategy terminates by building a separated ribbon: there is no Zed rule to apply to build a new BExp redex, so one can continue the strategy preserving reachability, or we have already a separated ribbon.

Corollary 3. The reachability problem for ribbon between states reduces to the reachability problem for separated ribbon between states.

We easily extend the lemma 6 to ribbons from $\{s\}$ to an arbitrary set $T$ : the implemented separation strategy both terminates and normalizes in a separated ribbon from $\{s\}$ to $T$ (extended as expected) preserving the reachability.

This is generalizable to separation for ribbons between arbitrary sets when there is a bridge between $S$ and $T$ (Definition 4). We are interested in bridged ribbons because they are always associated to a positive promenade in a BVASS.

Lemma 7. Let $V$ be a bridged ribbon from $S$ to $T$. Let $\mathcal{V}=\left(m, \mathcal{R}_{2}\right)$ be a rewriting system. There is a separating strategy for $\mathcal{V}$ rewriting $V$ into a separated ribbon $W$ from $S$ to $T$ such that: $\quad S^{\prime} \rightsquigarrow_{V} T^{\prime} \quad \Leftrightarrow \quad S^{\prime} \rightsquigarrow W T^{\prime}$.

Proof. Let $\rho$ be a ribbon from $S$ to $T$ with a bridge such that the, at most three, simply connected components are denoted by $V_{i}^{\rho}, i \in I$. Let $i \in I . V_{i}^{\rho}$ is by definition a ribbon from $S_{i}$ to $T_{i}$ where either $S_{i}$ or $T_{i}$ is a singleton, whose state is a state of the bridge. W.l.g. let $S_{i}=\left\{s_{i}\right\}$ be an arbitrary such singleton. By the previous extension of lemma $7, V_{i}^{\rho}$ is separable in $W_{i}^{\rho}$ between the same sets such that reachability is preserved. Let $W$ be the normal form by separation strategy of the ribbon which consists of the bridge of $\rho$ added to the $W_{i}^{\rho}$ ribbon. We have that $W$ is the normal form of $\rho$ and again reachability is preserved.

Remark that there is a bridge in all ribbons from $S$ to $T$ if at least one of these sets is a singleton. In fact one bridge is the arc or arc pair which is to or from the state of the singleton set. So bridged ribbons generalize ribbons from a singleton to a set.

To compare bridged ribbon reachability to VASS reachability, we want to reduce reachability of arbitrary separated ribbon to VASS reachability. We have:

Lemma 8. Let $\rho$ be a separated ribbon between arbitrary sets $S$ and $T$. Let $s$ and $t$ be two states not in $\rho$. Let $\theta_{s, S}$ (respectively $\theta_{T, t}$ ) be a VASP of same dimension than $\rho$ consisting of a binary tree of split pairs (respectively of join pairs) from $\{s\}$ to $S$ (respectively from $T$ to $\{t\}$ ). Let $W$ be the ribbon from $\{s\}$ to $\{t\}$ which consists of $\theta_{s, S}$ composed with $\rho$ and composed with $\theta_{T, t}$ (by 
identity morphism on $S$ and $T$ ). We have: if $x$ (respectively $y$ ) is a division of $\left\{x_{i}\right\}_{1 \leqslant i \leqslant|S|}$ (respectively of $\left\{y_{j}\right\}_{1 \leqslant i \leqslant|T|}$ ) then

$$
\left\{\left(s_{i}, x_{i}\right)_{s_{i} \in S}\right\} \rightsquigarrow \rho\left\{\left(t_{j}, y_{j}\right)_{t_{j} \in T}\right\} \quad \Leftrightarrow \quad\{(s, x)\} \rightsquigarrow W\{(t, y)\}
$$

Remark that the ribbon $W$ is separable, therefore there is a reduction between reachability for bridged ribbon and VASS reachability using a separability strategy. So by lemma 7 and 8 we have:

Corollary 4. The reachability problem for bridged ribbons reduces to the reachability problem for VASS (via many-one reductions). Then the former is decidable.

We finish a last step further with a strategy for VASP which are not ribbons: Repeat

rewrite a ribbon between arbitrary sets by separation strategy Until all ribbons are separated.

This (too strong) strategy does not always terminate, sometimes for bad reasons: rules cannot be applied because of interface restrictions, for example, when there is an arc to a node of the left-hand side of a rule, whose target or source is not in the interface. Rewriting rules with interfaces which consist of all the states of the left-hand side are quite inextricable (from a reachability point of view). Thus we are even far from semidecidability.

Lemma 9. Given a VASP $V$, if the separation strategy terminates for $V$ in a normal form $W$, we have: $\quad S \rightsquigarrow V T \Rightarrow S \rightsquigarrow W T$.

In such a normal form, all ribbons are separated. So by corollary 4 we have:

Proposition 2. Given a VASP $V$, if the separation strategy terminates for $V$, then the reachability problem for $V$ with divisible initial and final configurations reduces to VASS reachability problem.

\section{Conclusion}

We introduce a generalization of VASS called Vector Addition Systems with Pairs (VASP) by pairing arcs with same source or with same target. These correspond to split and joint transitions with a multiset of vectors. The reachability decision problem for VASP $\mathcal{R} \mathcal{P}_{\text {VASP }}$ subsumes the one for BVASS (as a sub-case of VASP without split pairs) which is equivalent to the open MELL provability decision problem. There is also a natural simplification of $\mathcal{R} \mathcal{P}_{\text {VASP }}$ not valid for BVASS.

We present graph rewriting systems in order to study paths in VASP. This tool permits reduction between restricted forms of VASP and VASS, preserving reachability properties. Notably the reachability problem is decidable for VASP in which our separation strategy terminates.

Other strategies, like zipping one using $\{$ RSum,BZip,BSwap,BCol $\}$ rules and reversed rules, can be used to obtain reachability for other kind of VASP. By zipping strategy we think to rewrite a ribbon starting from a source state 
and applying rules step-by-step on each outgoing arcs (source paired or not), making synchronization on each target paired state by reducing the remaining branch of ribbon before it.

The main other way for reachability decision is to adapt the original proof of reachability for VASS to VASP. It seems approachable to obtain decidability associated to Karp and Miller "trees" for VASP.

\section{References}

1. T. Brázdil, P. Jancar, and A. Kucera. Reachability games on extended vector addition systems with states. CoRR, abs/1002.2557, 2010.

2. P. de Groote, B. Guillaume, and S. Salvati. Vector addition tree automata. In LICS, pages 64-73. IEEE Computer Society, 2004.

3. S. Demri, M. Jurdzinski, O. Lachish, and R. Lazic. The covering and boundedness problems for branching vector addition systems. In R. Kannan and K. N. Kumar, editors, FSTTCS, volume 4 of LIPIcs, pages 181-192, 2009.

4. J. Esparza and M. Nielsen. Decidability issues for petri nets - a survey. Bulletin of the EATCS, 52:244-262, 1994.

5. G. Gallo, G. Longo, and S. Pallottino. Directed hypergraphs and applications. Discrete Applied Mathematics, 42(2):177-201, 1993.

6. S. Ginsburg and E. H. Spanier. Semigroups, presburger formulas, and languages. Pacific Journal of Mathematic, 16(2):285-296, 1966.

7. A. Ginzburg and M. Yoeli. Vector addition systems and regular languages. $J$. Comput. Syst. Sci., 20(3):277-284, 1980.

8. J.-Y. Girard. Linear logic. Theor. Comput. Sci., 50:1-102, 1987.

9. J. E. Hopcroft and J.-J. Pansiot. On the reachability problem for 5-dimensional vector addition systems. TCS, 8:135-159, 1979.

10. R. M. Karp and R. E. Miller. Parallel program schemata. J. Comput. Syst. Sci., 3(2):147-195, 1969.

11. S. R. Kosaraju. Decidability of reachability in vector addition systems (preliminary version). In STOC, pages 267-281. ACM, 1982.

12. J.-L. Lambert. A structure to decide reachability in petri nets. Theor. Comput. Sci., 99(1):79-104, 1992.

13. E. W. Mayr. An algorithm for the general petri net reachability problem. SIAM J. Comput., 13(3):441-460, 1984.

14. H. Müller. The reachability problem for vas. In G. Rozenberg, H. J. Genrich, and G. Roucairol, editors, European Workshop on App. and Theory in Petri Nets, volume 188 of Lecture Notes in Computer Science, pages 376-391. Springer, 1984.

15. R. Parikh. On context-free languages. J. ACM, 13(4):570-581, 1966.

16. D. Plump. Termination of graph rewriting is undecidable. Fundam. Inform., 33(2):201-209, 1998.

17. C. Reutenauer. Aspects Mathématiques des Réseaux de Pétri. Masson, 1989.

18. K. N. Verma and J. Goubault-Larrecq. Karp-miller trees for a branching extension of vass. Discrete Mathematics $\& 5$ Theoretical Computer Science, 7(1):217-230, 2005.

19. K. N. Verma and J. Goubault-Larrecq. Alternating two-way ac-tree automata. Inf. Comput., 205(6):817-869, 2007. 


\section{A Detailled Proofs}

We give the proofs of the lemmas 2, 3, 4 and 5 .

Lemma 2. Let $V$ be a inductive ribbon from $\{s\}$ to $\{t\}$ with valuation $v$. Let $W$ be a VASP of same dimension which is only a regular arc $\left(s^{\prime}, t^{\prime}\right)$ valued by $\Sigma v(a)$ for all regular arc $a \in V$. We have:

$$
\left\{\left(s^{\prime}, x\right)\right\} \rightsquigarrow W\left\{\left(t^{\prime}, y\right)\right\} \quad \Rightarrow \quad\{(s, x)\} \rightsquigarrow V\{(t, y)\} .
$$

Proof. We give a sketch where we consider inductive ribbon where there is one regular arc between two arcs of different pairs. Remark that by definition we have equality of the underlying binary subtree heights. General case is straightforward. Given $m>0$, we define an inductive ribbon $S$ of dimension $m$ inductively on what we call size $(S)$. Let an inductive ribbon of size 0 be a regular arc from $\left\{a_{0}\right\}$ to $\left\{b_{0}\right\}$. Let $S_{U, V}$ be an inductive ribbon of size $n$ from $\left\{a_{S}\right\}$ to $\left\{b_{S}\right\}$ consisting of a regular arc from $a_{S}$ to $a$, a ribbon $T_{U, V}$ from $\{a\}$ to $\{b\}$, and a regular arc from $b$ to $b_{S}$ such that $T_{U, V}$ consists of a sharing source $a$ with target states $a_{U}$ and $a_{V}$, a sharing target $b$ with source states $b_{U}$ and $b_{V}$, a regular arc from $b$ to $b_{S}$, and $U$ and $V$ two inductively defined separated ribbons respectively from $\left\{a_{U}\right\}$ to $\left\{b_{U}\right\}$ and from $\left\{a_{V}\right\}$ to $\left\{b_{V}\right\}$ such that $n=1+\max \{\operatorname{size}(U)$, size $(V)\}$.

We denote by $v$ the valuation function of any VASP when it is unambiguous. Given the previous $T_{U, V}$ from $\{a\}$ to $\{b\}$, we associate $R_{T}$ consisting of one regular $\operatorname{arc}\left(a^{\prime}, b^{\prime}\right)$ such that $\Sigma_{r \in R_{T}} v(r)=\Sigma_{r \in T_{U, V}} v(r)\left(\star_{1}\right)$. Given $S_{U, V}$ from $\left\{a_{S}\right\}$ to $\left\{b_{S}\right\}$, we associate $R_{S}$ consisting of three successive regular arcs: the first $\left(a_{S}^{\prime}, a^{\prime}\right)$ is isomorphic to $\left(a_{S}, a\right)$ with same valuation, the second is $R_{T}$ associated to $T_{U, V}$, the last $\left(b^{\prime}, b_{S}^{\prime}\right)$ is isomorphic to $\left(b, b_{S}\right)$ with same valuation.

Now we prove by strong induction on $\operatorname{size}(S)=n$ that $\forall n \geqslant 0$,

$$
\left\{\left(a_{S}^{\prime}, x\right)\right\} \rightsquigarrow R_{S}\left\{\left(b_{S}^{\prime}, y\right)\right\} \Rightarrow\left\{\left(a_{S}, x\right)\right\} \rightsquigarrow_{S}\left\{\left(b_{S}, y\right)\right\} .
$$

Base case is trivial: $S$ is isomorphic to $R_{S}$. For general case, let $S_{U, V}$ be a separated ribbon of size $n \geqslant 0$, and suppose that for all $m$ such that $n>m \geqslant 0$ the property holds $(H I)$.

First (boring) step: As $\left(a_{S}, a\right)$ and $\left(b, b_{S}\right)$ are the first and last arcs of $S_{U, V}$ with respectively same valuations $\left(v_{a}\right.$ and $\left.v_{b}\right)$ than first and last arcs of $R_{S}$, we have by definitions of $R_{S}$ and $S_{U, V}$ that $\left(\star_{2}\right)$ : for $X=x+v_{a}$ and $y=Y+v_{b}$,

$$
\begin{aligned}
& \left\{\left(a_{S}^{\prime}, x\right)\right\} \rightsquigarrow R_{S}\left\{\left(b_{S}^{\prime}, y\right)\right\} \Leftrightarrow\left\{\left(a^{\prime}, X\right)\right\} \rightsquigarrow R_{T}\left\{\left(b^{\prime}, Y\right)\right\} \\
& \text { and }\left\{\left(a_{S}, x\right)\right\} \rightsquigarrow_{S}\left\{\left(b_{S}, y\right)\right\} \Leftrightarrow\{(a, X)\} \rightsquigarrow_{T}\{(b, Y)\} \text {. }
\end{aligned}
$$

Inductive step: For all $\left(X_{1}, X_{2}\right)$ division of $X$, we have by $(H I)$ on both $U$ and $V$ :

$$
\begin{aligned}
& \left\{\left(a_{U}^{\prime}, X_{1}\right)\right\} \rightsquigarrow R_{U}\left\{\left(b_{U}^{\prime}, Y_{1}\right)\right\} \Rightarrow\left\{\left(a_{U}, X_{1}\right)\right\} \rightsquigarrow U\left\{\left(b_{U}, Y_{1}\right)\right\} \\
& \text { and }\left\{\left(a_{V}^{\prime}, X_{2}\right)\right\} \rightsquigarrow R_{V}\left\{\left(b_{V}^{\prime}, Y_{2}\right)\right\} \Rightarrow\left\{\left(a_{V}, X_{2}\right)\right\} \rightsquigarrow V\left\{\left(b_{V}, Y_{2}\right)\right\} \text {. }
\end{aligned}
$$

By $\left(\star_{1}\right)$, definitions of $T_{U, V}$ and $R_{T}$, and $(H I)$ on $U$ and $V$, we have:

If $\left(Y_{1}, Y_{2}\right)$ is a division of $Y$ then $\left\{\left(a^{\prime}, X\right)\right\} \rightsquigarrow R_{T}\left\{\left(b^{\prime}, Y\right)\right\} \Rightarrow\{(a, X)\} \rightsquigarrow_{T}\{(b, Y)\}$.

Finally by $\left(\star_{2}\right)$ we obtain the result. 
Lemma 3 (Key lemma). Given $m>0$, let $r=(m, L \supseteq K \subseteq R)$ be one of the previously defined rules except $Z$ ed but including reversed and inversed rules. Let $C$ be a set-configuration of zero in-degree states of $L$ (labelled $Q, Q^{\prime}$ for Backward rules in Fig.3). Let $C^{\prime}$ be a set-configuration of zero out-degree states of $R$. We have:

$$
C \rightsquigarrow_{L} C^{\prime} \quad \Leftrightarrow \quad C \rightsquigarrow_{R} C^{\prime}
$$

Proof. Let $r \in \mathcal{R}$. By definition $C$ and $C^{\prime}$ are set-configurations of the interface of rule $r$, then they are set-configurations of $R$. By case analysis we have:

- The result is obvious for BSwap and Col, and it is obtained by valuation definition for rule $R S u m$,

- For the BZip rule, the right implication is clear, but for the converse let denote by $c$ the regular arc of $R$, and $a$ and $b$ the regular $\operatorname{arcs}$ of $L$. By definition of BZip we have a divided valuation: $(v(a), v(b))$ is a division of $v(c)$. Let $C=\{(Q, x)\}$, if $C \rightsquigarrow R C^{\prime}$ then $x \geqslant v(c)$ in $R$, and for any division $\left(x_{1}, x_{2}\right)$ of $x-v(c)$ we have $C^{\prime}=\left\{\left(R, x_{1}\right),\left(S, x_{2}\right)\right\}$. By lemma 1 let take a division $\left(x_{1}, x_{2}\right)$ of $x-v(c)$ such that $x_{1} \geqslant v(a)$ and $x_{2} \geqslant v(b)$. This is the requirement for reachability from $C$ in $L$ : we obtain $C \rightsquigarrow_{L} C^{\prime} \Leftarrow C \rightsquigarrow{ }_{R} C^{\prime}$,

- For the sub-case rule BExp without regular arcs, we have $C^{\prime}=\left\{(R, y),\left(R^{\prime}, y^{\prime}\right)\right\}$ is reachable in $L_{B E x p}$ from $C=\left\{(Q, x),\left(Q^{\prime}, x^{\prime}\right)\right\}$ for any division $\left(y, y^{\prime}\right)$ of $x+x^{\prime}$. On the other side, we have for any division $\left(x_{1}, x_{2}\right)$ of $x$, and $\left(x_{1}^{\prime}, x_{2}^{\prime}\right)$ of $x^{\prime}$ that $C^{\prime}=\left\{\left(R, x_{1}+x_{1}^{\prime}\right),\left(R^{\prime}, x_{2}+x_{2}^{\prime}\right)\right\}$ is reachable in $R_{B E x p}$ from $C=\left\{(Q, x),\left(Q^{\prime}, x^{\prime}\right)\right\}$. For any division $\left(x_{1}, x_{2}\right)$ of $x$ and $\left(x_{1}^{\prime}, x^{\prime} 2\right)$ of $x^{\prime}$, we have that $\left(x_{1}+x_{1}^{\prime}, x_{2}+x_{2}^{\prime}\right)$ is a division of $x+x^{\prime}$, then $C \rightsquigarrow_{L} C^{\prime} \Leftarrow C \rightsquigarrow_{R} C^{\prime}$. Conversely, for any division of $x+x^{\prime}$ there is two divisions of $x$ and $x^{\prime}$ whose component-wise sum corresponds to $\left(y, y^{\prime}\right)$, then $C \rightsquigarrow_{L} C^{\prime} \Rightarrow C \rightsquigarrow_{R} C^{\prime}$,

- For the rule BExp with regular arcs. Remark that the left-hand side of $B E x p$ can be rewritten by $B Z i p^{-1}$ rule: the result contains a redex of $B E x p$ without regular arc. For $B Z i p^{-1}$ we have seen that $C \rightsquigarrow_{R} C^{\prime} \Leftrightarrow C \rightsquigarrow_{L} C^{\prime}$, and for BExp without regular arc, we have also that $C \rightsquigarrow_{L} C^{\prime} \Leftrightarrow C \rightsquigarrow_{R} C^{\prime}$. Then the results holds,

- For reversed Backward rules, we have the results for the same reason than corresponding rule, because of a division of a source sharing pair is the "reverse" of a division of a target sharing pair, and reciprocally. For inverse rules, by the equivalences proven the result holds.

Lemma 4. Given a rewriting system $(m,\{B E x p\})$, there is no infinite sequence of rewrite steps in a ribbon.

Proof. We give a sketch with hyperarcs: in this case we have a measure that decreases in every BExp redex context, so terminaison for this rewrite rule generalized to hyperarcs. This give us a bound on the number of BExp rewrite steps in the VASP by simulating the corresponding rule for hyperarcs with fixed steps.

Let $V$ be a ribbon, and let $V^{\prime}$ the corresponding VAHA where all hyperarcs have one source or one target. Given $M$ a maximal alternating sequence of a 
sharing target arc pair followed by an sharing source arc pair in $V^{\prime}$. Let $n$ be the length of $M$. Let $B E X P$ be the set of rules generalizing the $B E x p$ rule without regular arcs to arbitrary VAHA hyperarc arities. The interface of $B E X P$ is a set $\left\{s_{i}\right\}_{i \in I} \uplus\left\{t_{j}\right\}_{j \in J}$ and the left-hand side contains one more state $p$ such that $p$ is a shared target of arcs from $\left\{s_{i}\right\}_{i \in I}$, and $p$ is a shared source of arcs to $\left\{t_{j}\right\}_{j \in J}$. In the right-hand side, the $s_{i}$ for $i \in I$ are each shared sources, and the $t_{j}$ for $j \in J$ are each shared targets. Applying BEXP to $M$ strictly decreases $n$ (even if the result has interactions with the context) du to the fact that we use isomorphism between two consecutive hyperarcs with source shared and one hyperarc with source shared but greater arity. This ensures terminaison of BEXP rewriting. As the maximal arity of $B E X P$ rules needed to obtain the normal form of $V^{\prime}$ is fixed, one can simulate each $B E X P$ rewrite steps by a fixed number of $B E x p$ rewrite steps in $V$.

Lemma 5. Let $V$ be a separated ribbon from $\{s\}$ to $\{t\}$ with valuation $v$. Let $W$ be a VASP of same dimension which is only a regular arc $\left(s^{\prime}, t^{\prime}\right)$ valued by $\Sigma v(a)$ for all regular arc $a \in V$. We have:

$$
\{(s, x)\} \rightsquigarrow V\{(t, y)\} \quad \Leftrightarrow \quad\left\{\left(s^{\prime}, x\right)\right\} \rightsquigarrow W\left\{\left(t^{\prime}, y\right)\right\}
$$

Proof. Let $V$ be a separated ribbon from $\{s\}$ to $\{t\}$ of dimension $m>0$ and valuation $v$. Let $W$ be the regular arc defined in the lemma hypothesis. Let $\mathcal{V}=(m,\{R S u m, C o l, B Z i p, F Z i p\})$ be a rewriting system. Let $U$ be the normal form of $V$ obtained by the following strategy on $\mathcal{V}$ : firstly from $s$ we apply $\overrightarrow{\mathcal{F}}=\left(\rightarrow_{\text {RSum }}^{*} \rightarrow_{B Z i p}\right)^{*}$ on the 2 -tree with root $\{s\}$, and we apply from $t$ the same reversed strategy, that is $\overleftarrow{\mathcal{F}}=\left(\rightarrow_{\text {RSum }}^{*} \rightarrow_{F Z i p}\right)^{*}$, on the reversed 2-tree with root $\{t\}$. Secondly we apply the strategy $\mathcal{G}=\rightarrow_{\text {Col }}^{*}$ and we finish by the strategy $\mathcal{H}=\rightarrow_{\text {RSum }}^{*}$.

By definition the strategy $\overrightarrow{\mathcal{F}}$ is (strongly) normalizing on 2-trees. The normal form of $\overrightarrow{\mathcal{F}}$ is a sequence of regular arcs that finishes by $B_{1}$ a binary tree of shared source pairs with leave set isomorphic to the one of the original 2-tree. Remark that the reversed strategy $\overleftarrow{\mathcal{F}}$ has the same properties than $\overrightarrow{\mathcal{F}}$ because of they are preserved by reversing. Let $B_{2}$ be the binary tree obtained.

At second time, we apply $\mathcal{G}$ strategy to the VASP $B$ consisting of $B_{1}$ and $B_{2}$ (which have the same set of leaves). Remark that for reachability the order of not paired states in a pair is irrelevant. Then by classical combinatoric arguments we have $B$ normalizes by $\mathcal{G}$ in one state merging all states of $B$.

At this time $V$ is normalized in a sequence of regular arcs which is rewritten in a single regular arc by strategy $\mathcal{H}$. This regular arc is from $f(s)$ to $f(t)$ for a trivial morphism $f$ such that its valuation is $\Sigma v(a)$ for all regular arc $a \in V$. In other words it is isomorphic to $W$, and then $V$ is normalized in $W$ by a strategy on $\mathcal{V}$. Considering rules in $\mathcal{V}$, we have the result by lemma 3 . 